\title{
Alteration of cytochrome oxidase subunit I labeling is associated with severe mitochondriopathy in NRTI-related hepatotoxicity in HIV patients
}

Jean-Paul Duong Van Huyen ${ }^{1,2}$, Dominique Batisse ${ }^{3}$, Didier Heudes ${ }^{1,2}$, Marie-France Belair ${ }^{2}$, Christophe Piketty ${ }^{3}$, Gustavo Gonzalez-Canali ${ }^{3}$, Laurence Weiss $^{3}$, Michel D Kazatchkine ${ }^{3}$ and Patrick Bruneval ${ }^{1,2}$

${ }^{1}$ Department of Pathology, Hôpital Européen Georges Pompidou, Université Paris V René Descartes, Paris, France; ${ }^{2}$ INSERM U652, Institut des Cordeliers, Paris, France and ${ }^{3}$ Department of Clinical Immunology, Hôpital Européen Georges Pompidou, Université Paris V René Descartes, Paris, France

Liver mitochondrial toxicity induced by nucleoside reverse transcriptase inhibitors (NRTI) in human immunodeficiency virus (HIV) patients has been associated with a wide range of liver involvement ranging from low-grade hepatotoxicity, asymptomatic lactacidemia to severe liver insufficiency, with massive steatosis and life-threatening lactic acidosis. Considerable efforts have been made in the last few years to establish clinical guidelines to avoid life-threatening NRTI-associated lactic acidosis. However, the important issue of low-grade NRTI-associated hepatotoxicity still needs to be unravelled since its natural history is largely unknown. We have recently reported a series of 13 monoinfected HIV patients with low-grade NRTI-associated toxicity. Our results outlined the heterogeneity of NRTI-induced hepatotoxicity and raised the question of its diagnosis. The present study evaluates the expression of cytochrome oxidase (COX) subunits I and IV, encoded by mitochondrial and nuclear DNA, respectively, in NRTI hepatotoxicity. The aim of our study was to compare the detection rate of mitochondrial abnormalities of immunohistochemistry for COX subunit I with electron microscopy. COX subunit I and IV labeling was performed together with light microscopy and ultrastructural analysis in a series of 55 liver biopsies from HIV monoinfected and HIV-hepatitis C virus coinfected patients. Clinical data were also recorded. Our major findings were: (i) decreased COX subunit I labeling is associated with severe ultrastructural mitochondrial alterations and may represent overt NRTI-induced mitochondrial cytopathy; (ii) mild ultrastructural damage associated with normal COX subunit I labeling is of unknown clinical significance. The results of the study suggest that COX subunit I labeling may be a valuable tool for the diagnosis of mitochondrial liver disease in HIV patients.

Modern Pathology (2006) 19, 1277-1288. doi:10.1038/modpathol.3800652; published online 30 June 2006

Keywords: nucleoside reverse transcriptase inhibitor; liver biopsy; electron microscopy; immunohistochemistry; mitochondrial toxicity

The benefits of antiretroviral combined therapy in human immunodeficiency virus (HIV)-infected patients in terms of mortality and morbidity have been clearly established. ${ }^{1}$ However, the increase in survival due to the extensive use of highly active antiretroviral therapy in HIV patients is associated

Correspondence: Dr J-P Duong Van Huyen, Laboratoire d'Anatomie Pathologique, Hôpital Européen Georges Pompidou, 20 rue Leblanc, 75908 Paris, Cedex 15, France.

E-mail: jean-paul.duong-van-huyen@hop.egp.ap-hop-paris.fr Received 7 March 2006; revised and accepted 31 May 2006; published online 30 June 2006 with the emergence of a number of pathological conditions. Among them, liver diseases are of importance with a dramatic raise of associated-liver morbidity and mortality within the last decade, ${ }^{2}$ mainly attributed to chronic hepatitis $\mathrm{C}$ virus (HCV) coinfection, ${ }^{3-5}$ and to liver toxicities of antiretroviral drugs. ${ }^{6}$ The major liver adverse event of HAART is the nucleoside reverse transcriptase inhibitors (NRTI)-induced liver mitochondriopathy that is related to defective mitochondrial DNA (mtDNA) replication due to the NRTI inhibition of the mtDNA polymerase $\gamma^{7,8}$ The impairment of mitochondrial functions in the liver results in a cascade of 
deleterious events including the release of cellular lactate and defective liver fatty acid $\beta$-oxidation leading to steatosis. NRTI-associated liver toxicity ranges from potentially lethal lactic acidosis with massive steatosis and liver insufficiency to asymptomatic lactacidemia and/or mild raise in liver enzymes. ${ }^{6,7,9}$ Considerable efforts have been made the last few years to establish guidelines to avoid life-threatening NRTI-associated lactic acidosis. ${ }^{6}$ In contrast, low-grade NRTI hepatotoxicity (grade 1-2 hepatotoxicity defined as nonsymptomatic patients with aminotransferase (AT) below $5 \mathrm{~N}$ and without lactacidemia) still needs to be unravelled since its natural history is largely unknown. Thus, it is currently not know if it represents a factor of cumulative chronic liver impairment, together with chronic hepatitis $\mathrm{C}$ or alcoholic liver disease.

We recently described the liver histology and electron microscopy (EM) findings in a series of monoinfected HIV patients treated with NRTI-based regimens. ${ }^{10}$ In this study, we observed that clinically and biologically defined low-grade hepatotoxicity could be associated with severe ultrastructural mitochondrial impairment and inconstant steatosis. Our results outlined the heterogeneity of NRTIinduced low-grade hepatotoxicity and raised the question of its diagnosis. ${ }^{10,11}$ Therefore EM that has been considered as the gold standard in the diagnosis of mitochondrial cytopathy may be limited in this particular field of low-grade hepatotoxicity. This issue, together with other limitations of EM, that is,the low tissue sampling and the rare availability of EM for routine practice in the majority of the centers, emphasizes the need to address complementary markers for the diagnosis of NRTI hepatotoxicity. New biochemical and molecular approaches have been recently proposed for the diagnosis of NRTI mitochondriopathy, but need to be validated in clinical practice. ${ }^{12-15}$ In this regard, various antibodies raised against proteins involved in mitochondrial oxidative phosphorylation are now available for immunohistochemistry on formalin-fixed paraffin-embedded tissues. ${ }^{16}$

In the present study, the immunohistochemical detection of respiratory chain protein cytochrome oxidase (COX) subunits I and IV, encoded by mtDNA and nuclear DNA (nDNA), respectively, was performed in liver biopsies from HIV patients. The aim of our study was to compare the detection rate of mitochondrial abnormalities of immunohistochemistry for COX subunit I with electron microscopy. Our results suggest the validity of immunohistochemical analysis of COX subunits for the diagnosis of liver NRTI-related mitochondriopathy.

\section{Materials and methods}

\section{Patients}

In this study, all the cases were HIV-seropositive patients followed in the department of Clinical
Immunology of the Hôpital Européen Georges Pompidou in Paris, France. All patients underwent a liver biopsy with both histological and ultrastructural assessment. One group included 16 HIV monoinfected patients retrieved retrospectively from our files, all being treated with NRTI-based regimen. Liver biopsy was performed in these 16 monoinfected patients because of suspicion of liver toxicity. Some of these patients have already been reported in our previous study. ${ }^{10}$ A second group included 39 coinfected HIV-HCV patients that were enrolled prospectively in this study during a period of 2 years. Patients were biopsied either for the follow-up of a chronic hepatitis $\mathrm{C}$ or for unexpected abnormalities of liver enzymes. Nine HIV-HCV coinfected patients did not receive any antiretroviral treatment. Patients with liver insufficiency and/or lactic acidosis were not included in this study, because in these cases the diagnosis of severe NRTI liver toxicity did not require a liver biopsy with EM. Finally, because of the difference in the selection methods between HIV and HIV-HCV patients enrolled in this study, no statistical comparison was drawn between these two groups.

For all the patients both clinical and biological data at the time of the liver biopsy were recorded. Laboratory tests included liver tests, that is, serum AT AST (normal range 9-36 IU/l) and ALT (normal range $7-35 \mathrm{IU} / \mathrm{l}$ ), alkaline phosphatase (AP, normal range 30-80 IU/l) and gamma glutamyl transferase ( $\gamma \mathrm{GT}$ : normal range 10-45 IU/l), serum lactates (normal $<2.2 \mathrm{mmol} / \mathrm{l}$ ), serum lipid triglycerides (normal range $0.45-1.70 \mathrm{mmol} / \mathrm{l}$ ), and total cholesterol (normal range $4-5.2 \mathrm{mmol} / \mathrm{l}$ ), creatine phosphate kinase (CPK, normal range 15-140 IU/l), and lipase (normal range 7-60IU/1). An extrahepatic syndrome was defined by either neuromuscular, digestive or pancreatic clinical or biological abnormalities, that is muscular weakness, peripheral neuropathy, digestive symptoms such as diarrhea with weight loss, significant and durable rise of CPK or lipase. In the absence of any other cause, these extrahepatic symptoms were considered as suggestive of antiretroviral toxicity. ${ }^{7}$

\section{Tissue Sampling and Histopathology}

Liver percutaneous needle biopsies were cut into two pieces, one piece ( $>2 \mathrm{~mm}$ long) was immediately fixed in $2.5 \%$ glutaraldehyde for EM and the other (>10 mm long) in $10 \%$ buffered formalin for standard histology. Paraffin sections were stained with hematoxylin-eosin, picrosirius red and Perl's method. Sections were evaluated by two different observers (JPDVH and PB) for steatosis, hepatocyte ballooning, Mallory's hyaline, megamitochondria, portal and lobular inflammation and fibrosis. Overall steatosis, including macrovacuolar and microvesicular steatosis, was graded (St-g) according to the semiquantitative score established by Rullier 
et $a l^{5}$ (grade 0: none, grade 1: up to $10 \%$, grade 2: $10-30 \%$, grade $3:>30 \%$ ). In addition, foci of microvesicular steatosis were recorded when present. In coinfected patients, periportal inflammation (0-3) and intralobular necrosis (0-2) were graded using the METAVIR scoring system, ${ }^{17}$ with the activity algorithm (A). Portal fibrosis (0-4) was also defined and graded according to the METAVIR scoring system. In addition, the presence of perisinusoidal fibrosis (0-1) within the lobule was assessed on Sirius red stained sections.

\section{Electron Microscopy}

Glutaraldehyde-fixed samples were available for all patients and were processed following standard EM technique as previously described. ${ }^{10,11}$ Mitochondial abnormalities were defined by alterations of mitochondrial profiles and by the presence of mitochondrial inclusions. Mitochondrial abnormalities were semiquantitatively evaluated according to the extent of mitochondrial damage: grade 1 (Mt-g1= mild) was defined by a partial loss of mitochondrial profiles; grade 2 (Mt-g2= severe) included total or subtotal loss of mitochondrial profiles. Ultrastructural grading of mitochondrial abnormalities was performed by two observers (JPDVH and PB) in a blinded fashion.

\section{Immunohistochemistry}

Immunohistochemical studies were performed with antibodies specific for respiratory chain proteins that are encoded by mtDNA or by nDNA. Mouse monoclonal antibodies (Molecular Probes Inc, Eugene, OR, USA) raised against human COX subunit I (clone 1D6) which is mtDNA encoded and human COX subunit IV (clone 20E8), which is nDNA encoded were used. ${ }^{16}$ A three step avidinbiotin immunoperoxidase method with prior heatinduced antigen retrieval (TRS, Dako, Trappes, France) was performed on formalin-fixed paraffinembedded liver biopsy sections. Labeling of COX subunits was assessed by three observers (JPDVH, $\mathrm{MFB}$, and $\mathrm{PB}$ ) in a blinded fashion. Control tissue samples were obtained from normal area from surgical hepatectomy for cancer or from liver biopsies of patients with chronic viral hepatitis or alcoholic liver disease.

\section{Statistic Analysis}

Results were expressed as mean + / - standard error. Comparison of quantitative variables was performed using ANOVA and Mann-Whitney test. $\chi^{2}$ test was used to compare qualitative and semiquantitative variables. For small numbers statistics, Yate's correction was used after appropriate clustering of cases. All analyses were performed using Statview ${ }^{\mathbb{R}}$ software. Statistical significance was set at $P<0.05$.

\section{Results}

\section{Clinical Findings}

The characteristics of the patients at the time of the liver biopsy are summarized in Table 1. In our series, they were 47 male and eight female patients with a median age of 43 years (range 33-56 years). Median CD4 count and HIV RNA at the time of the liver biopsy were $462 \times 10^{6} / \mathrm{l}$ (range 52-1400 $\times 10^{6} / \mathrm{l}$ ), and $2.88 \mathrm{log}$ copies/ml (range 1.70-5.38 log copies/ml), respectively. The median duration time of HIV infection was 11 years (range $2-23$ years). No patient had any present history of alcohol abuse. Circulating hepatitis B surface antigen was absent in all cases. None of these patients was treated with other mitochondrial damaging drugs, that is, valproate, tetracyclins.

All HIV monoinfected patients were treated with NRTI alone or with an association of two NRTI with non-nucleoside reverse transcriptase inhibitors (NNRTI) and/or protease inhibitors (PI). Abnormalities of the liver tests were constantly present in the HIV monoinfected group. Mean lactates were $2.5 \mathrm{mmol} / \mathrm{l}$ without any acidosis. Extrahepatic syndrome suggestive of antiretroviral toxicity was present in nine patients. It was characterized by neuromuscular abnormalities, digestive symptoms with weight loss, elevated lipase, and CPK.

In the HIV-HCV coinfected group, nine patients were untreated and six patients received NRTI alone. Ten and 12 patients were treated with NRTI plus NNRTI and NRTI plus PI, respectively. Two patients received a triple association of antiretroviral drugs. All coinfected patients presented with elevated liver enzymes. Although mean lactates were normal, a mild increase in serum lactates $(>2.2 \mathrm{mmol} / \mathrm{l})$ was found in four patients without any acidosis. Extrahepatic syndrome was present in 15 cases.

\section{Histopathology}

Histopathology is illustrated in Figure 1 and summarized in Table 2.

\section{Steatosis and other hepatocyte changes}

Steatosis $\geq$ St-g1 was observed in 13 monoinfected patients $(81 \%)$. Mild steatosis (St-g1) and moderate to marked steatosis grades (St-g2 + St-g3) (Figure 1a), were present in 25 and $56 \%$ of the patients, respectively. In addition to macrovacuolar steatosis, foci of microvesicular steatosis were observed in five cases (Figure 1b). Globular megamitochondria were present in two cases (Figure 1c). Foci of ballooned hepatocytes (Figure 1d) and occasional Mallory bodies were present in 10 cases and four cases, respectively.

In HIV-HCV coinfected patients, mild steatosis (St-g1) and moderate to marked steatosis (St-g2 + Stg3) involved 49 and $15 \%$ of cases, respectively. The 
Table 1 Characteristics of patients at the time of liver biopsy

\begin{tabular}{|c|c|c|c|c|}
\hline Characteristics of the patients & HIV treated $(\mathrm{N}=16)$ & $H I V-H C V$ treated $(\mathrm{N}=30)$ & $H I V-H C V$ untreated $(\mathrm{N}=9)$ & $\mathrm{P}$ \\
\hline Male gender: $N(\%)$ & $14(87.5)$ & $28(93.3)$ & $5(55.5)$ & $<0.05$ \\
\hline Age (years): mean (range) & $47(35-57)$ & $41(33-54)$ & $42(33-51)$ & NS \\
\hline HIV duration (years): mean (range) & $9(2-18)$ & $12(3-21)$ & $13(4-23)$ & NS \\
\hline CD4 count (cells/mm³): mean (range) & $417(173-834)$ & $531(52-1400)$ & $420(266-964)$ & NS \\
\hline HIV RNA (log copies/ml): mean (range) & $2.94(1.70-4.95)$ & $2.38(1.40-4.71)$ & $4.29(2.99-5.38)$ & $<0.001$ \\
\hline \multicolumn{5}{|l|}{ Antiretroviral therapy: $N(\%)$} \\
\hline None & $0(0)$ & $0(0)$ & $9(100)$ & ND \\
\hline NRTI alone & $4(25)$ & $6(20)$ & $0(0)$ & \\
\hline NRTI+NNRTI & $7(43.7)$ & $10(33.3)$ & $0(0)$ & \\
\hline NRTI+PI & $4(25)$ & $12(40)$ & $0(0)$ & \\
\hline NRTI+NNRTI+PI & $1(6.3)$ & $2(6.7)$ & $0(0)$ & \\
\hline \multicolumn{5}{|l|}{ HCV genotype: $N(\%)^{\mathrm{a}}$} \\
\hline $1,1 \mathrm{a}, 1 \mathrm{~b}$ & ND & $18(60)$ & $5(55.5)$ & NS \\
\hline 2 & ND & 0 & 0 & \\
\hline 3 & ND & $7(23.3)$ & $2(22.2)$ & \\
\hline $4 \mathrm{c}, 4 \mathrm{~d}$ & ND & $3(10)$ & $1(11.1)$ & \\
\hline \multicolumn{5}{|l|}{ Biology } \\
\hline AST (IU/l): mean \pm s.e.m. & $71 \pm 8$ & $110 \pm 42$ & $55 \pm 11$ & NS \\
\hline ALT (IU/l): mean \pm s.e.m. & $104 \pm 17$ & $129 \pm 38$ & $62 \pm 17$ & NS \\
\hline AP (IU/l): mean \pm s.e.m. & $100 \pm 20$ & $74 \pm 3$ & $62 \pm 3$ & NS \\
\hline gGT (IU/l): mean \pm s.e.m. & $237 \pm 52$ & $157 \pm 30$ & $70 \pm 17$ & NS \\
\hline Lactates $(\mathrm{mmol} / \mathrm{l})$ : mean \pm s.e.m. & $2.5 \pm 0.3$ & $1.7 \pm 0.2$ & $1.2 \pm 0.3$ & NS \\
\hline Lipase (IU/l): mean \pm s.e.m. & $42 \pm 6$ & $38 \pm 4$ & $33 \pm 5$ & NS \\
\hline CPK (IU/l): mean \pm s.e.m. & $154 \pm 37$ & $200 \pm 43$ & $98 \pm 11$ & NS \\
\hline Triglyceride (mmol/l): mean \pm s.e.m. & $2.8 \pm 0.7$ & $2.3 \pm 0.2$ & $1.3 \pm 0.2$ & $<0.05$ \\
\hline Total cholesterol (mmol/l): mean \pm s.e.m. & $5.1 \pm 0.3$ & $4.8 \pm 0.2$ & $4.1 \pm 0.1$ & NS \\
\hline
\end{tabular}

${ }^{\mathrm{a}} \mathrm{HCV}$ genotype was no evaluated in three patients.

AST: aspartate aminotransferase, ALT: alanine aminotransferase, AP: alkalin phosphatase, $\gamma \mathrm{GT}$ : gamma glutamyl transferase, CPK: creatine phosphate kinase.

Statistical anlysis with comparison between HIV-HCV treated vs HIV-HCV untreated groups.

mean steatosis grade was 0.79. Microvesicular steatoses were observed in two cases.

\section{Inflammation and fibrosis-Chronic hepatitis grading}

Both inflammation and fibrosis were semiquantitatively assessed in HIV monoinfected patients. Low-grade periportal activity and lobular necrosis were observed in 19 and $56 \%$ of these patients, respectively. Lobular activity consisted of acidophil necrosis or Councilman bodies with only minor inflammation, that is, rare lymphocytes and polymorphonuclears. Sixty-nine percent of monoinfected patients also exhibited a slight portal fibrosis associated with mild portal inflammation in less than one-third of the cases. Using Sirius red staining, perisinusoidal fibrosis was observed in $37 \%$ of monoinfected patients, mainly in cases with lobular activity.

Liver biopsies from HIV-HCV coinfected patients were graded according to the METAVIR scoring system. The activity and the fibrosis scores were $1.5+/-0.1$ and $1.7+/-0.2$, respectively. Onehalf of the coinfected patients had perisinusoidal fibrosis.

\section{Electron Microscopy}

EM is illustrated in Figure 2 and summarized in Table 2.

Mitochondrial abnormalities consisted of reduction to complete loss of mitochondrial profiles (Figure 2b-d). Paracristallin inclusions were found in 10 patients, sometimes in megamitochondria (Figure 2c). Autophagosomes containing remnants of degenerative mitochondria were also present. In addition, cholestasis and sinusoidal fibrosis, lipid droplets, were frequently observed (data not shown).

EM abnormalities are presented in Table 2. Among the 16 monoinfected patients, 15 had mitochondrial abnormalities, ranging from mild to severe (Mt-g1 $=6$ patients, Mt-g2 $=9$ patients) and one had normal mitochondria (Figure 2a). Thus, severe mitochondrial defects were observed in $56.2 \%$ of the monoinfected patients.

Among the 39 HIV-HCV coinfected patients, 27 had mitochondrial abnormalities (Mt-g1 $=22$ patients, Mt-g2 $=5$ patients). However, severe mitochondrial defects were present in only $12.8 \%$ of the coinfected patients. Interestingly, among the 12 coinfected patients without mitochondrial abnormalities, five were untreated and seven 

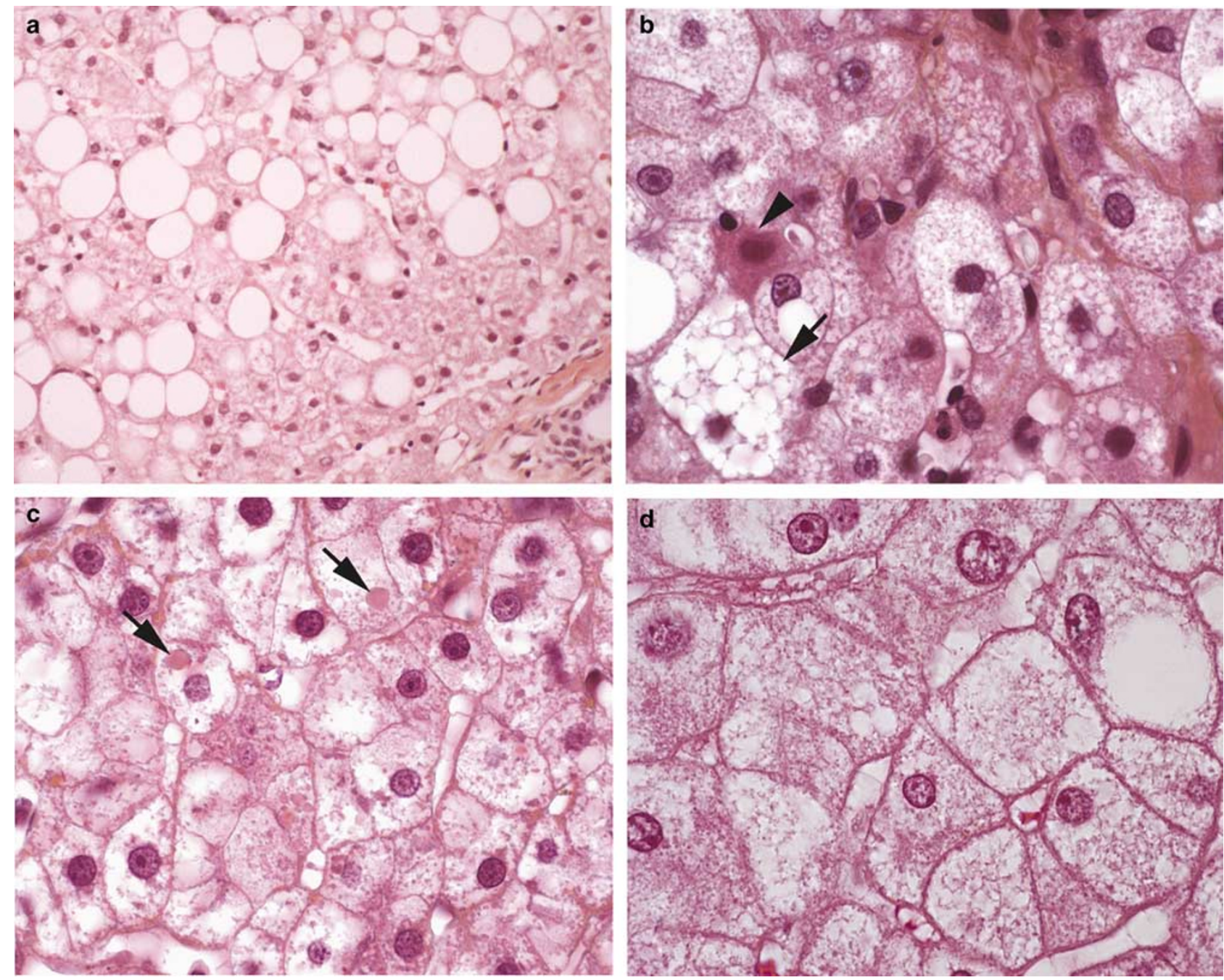

Figure 1 Histopathological findings in liver biopsies of HIV patients treated with NRTI-based regimen. (a) Grade St-g3 (severe, $>30 \%$ of the hepatocytes) macrovacuolar steatosis in a monoinfected patient. (b) Focus of microvesicular steatosis (arrow) in a monoinfected patient. Note the hepatocyte necrosis (arrowhead) with sparse inflammatory cells. (c) Megamitochondria (arrows) in hepatocytes of a monoinfected patient. (d) Area of hepatocyte ballooning degeneration in a HIV-HCV coinfected patient.

received NRTI-based regimen. Conversely, among the nine coinfected patients that were not treated by antiretroviral therapy, four presented mild (Mt-g1) ultrastructural mitochondrial abnormalities, and five had no ultrastructural abnormalities.

\section{Immunohistochemistry of COX Subunits I and IV}

COX subunit labeling is illustrated in Figures 3, 4 and summarized in Table 2 .

In control liver tissues

COX subunit I and IV immunostaining was first assessed in control percutaneous or surgical liver biopsies. A total of 15 tissue samples from normal liver, alcoholic liver disease, B and C viral hepatitis, all with various extent of inflammation and fibrosis were tested for COX subunits I and IV labeling. In all cases, a strong cytoplasmic immunoreactivity was present in hepatocytes and bile duct cells with both antibodies (Figure 3a-b). The hepatocytes were homogeneously labeled, with rare limited areas of slightly decreased staining (Figure 3c), mainly in the centrilobular zone. Interestingly, COX subunit labeling was unaffected by fibrosis or inflammation in both alcoholic disease or viral hepatitis. Thus, COX subunit staining was still homogenous in overt cirrhosis (Figure 3d).

\section{In liver biopsies of patients}

Decreased expression of COX subunit I labeling was observed in nine monoinfected and in five coinfected patients. In these cases, the loss of COX subunit I was variable between hepatocytes without any generalization resulting in a heterogenous and 
Table 2 Histopathology, electron microscopy and COX subunit I labeling

\begin{tabular}{|c|c|c|c|c|}
\hline & $\begin{array}{c}\text { HIV treated } \\
\mathrm{N}=16\end{array}$ & $\begin{array}{c}H I V-H C V \text { treated } \\
\mathrm{N}=30\end{array}$ & $\begin{array}{c}H I V-H C V \text { untreated } \\
\mathrm{N}=9\end{array}$ & $\mathrm{P}$ \\
\hline \multicolumn{5}{|l|}{ Histological findings } \\
\hline \multicolumn{5}{|l|}{ Steatosis: $N(\%)$} \\
\hline St-g0 & $3(18.7)$ & $10(33.3)$ & $4(44.4)$ & $\mathrm{NS}^{\mathrm{a}}$ \\
\hline St-g1 & $4(25)$ & $14(46.7)$ & $5(55.6)$ & \\
\hline St-g2 & $3(18.7)$ & $6(20)$ & $0(0)$ & \\
\hline St-g3 & $6(37.5)$ & $0(0)$ & $0(0)$ & \\
\hline Steatosis grade + s.e.m. & $1.7+0.3$ & $0.9+1.4$ & $0.6+1.8$ & NS \\
\hline Microvesicular statosis: $N(\%)$ & $5(\overline{3} 1.2)$ & $2(\overline{5})$ & $0(\overline{0})$ & NS \\
\hline Hepatocyte balloonning degeneration: $N(\%)$ & $10(62.5)$ & $8(26.7)$ & $0(0)$ & NS \\
\hline Megamitochondria: $N(\%)$ & $2(12.5)$ & $5(16.7)$ & $0(0)$ & NS \\
\hline Mallory bodies: $N(\%)$ & $4(25)$ & $1(3.4)$ & $0(0)$ & NS \\
\hline \multicolumn{5}{|l|}{ Periportal activity: N (\%) } \\
\hline 0 & $13(81.2)$ & $2(6.7)$ & $1(11.1)$ & $\mathrm{NS}^{\mathrm{b}}$ \\
\hline 1 & 3 (18.7) & $19(63.3)$ & $4(44.4)$ & \\
\hline 2 & 0 & $6(20)$ & $2(22.2)$ & \\
\hline 3 & 0 & $3(10)$ & $2(22.2)$ & \\
\hline \multicolumn{5}{|l|}{ Lobular necrosis: $N(\%)$} \\
\hline 0 & $7(43.7)$ & $7(23.3)$ & $4(44.4)$ & $\mathrm{NS}^{\mathrm{c}}$ \\
\hline 1 & $9(56.2)$ & $18(60)$ & $4(44.4)$ & \\
\hline 2 & 0 & $4(13.3)$ & $1(11.1)$ & \\
\hline Activity METAVIR (A): mean \pm s.e.m. & ND & $1.3 \pm 0.2$ & $1.5 \pm 0.2$ & NS \\
\hline \multicolumn{5}{|l|}{ Portal fibrosis (METAVIR F): N (\%) } \\
\hline 0 & $5(31.2)$ & $3(10)$ & $1(11.1)$ & $\mathrm{NS}^{\mathrm{d}}$ \\
\hline 1 & $11(68.7)$ & $12(40)$ & $4(44.4)$ & \\
\hline 2 & $0(0)$ & $7(23.3)$ & $3(33.3)$ & \\
\hline 3 & $0(0)$ & $4(13.3)$ & $1(11.1)$ & \\
\hline 4 & $0(0)$ & $4(13.3)$ & $0(0)$ & \\
\hline \multicolumn{5}{|l|}{ Perisinusoidal fibrosis: $N(\%)$} \\
\hline 0 & $10(62.5)$ & $13(43.3)$ & $7(77.8)$ & NS \\
\hline 1 & $6(37.5)$ & $17(56.7)$ & $2(22.2)$ & \\
\hline Fibrosis METAVIR (F): mean \pm s.e.m. & ND & $1.9 \pm 0.2$ & $1.4 \pm 0.3$ & NS \\
\hline \multicolumn{5}{|l|}{ Electron microscopy } \\
\hline Mt-g0: $N(\%)$ & $1(6.2)$ & 7 (23.3) & $5(55.6)$ & $\mathrm{NS}^{\mathrm{e}}$ \\
\hline Mt-g1: $N(\%)$ & $6(37.5)$ & $18(60)$ & $4(44.4)$ & \\
\hline Mt-g2: $N(\%)$ & $9(56.2)$ & $5(16.7)$ & $0(0)$ & \\
\hline EM grade: mean \pm s.e.m. & $1.5 \pm 0.2$ & $0.9 \pm 0.1$ & $0.4 \pm 0.2$ & $<0.05$ \\
\hline \multicolumn{5}{|l|}{ COX subunit I labeling } \\
\hline Normal: $N(\%)$ & $7(43.7)$ & $26(86.7)$ & $9(100)$ & NS \\
\hline Decreased: $N(\%)$ & $9(56.3)$ & 4 (13.3) & $0(0)$ & \\
\hline
\end{tabular}

Statistical analysis with comparison between HIV-HCV treated vs HIV-HCV untreated groups.

${ }^{\mathrm{a}} P$-value after clustering of St-g0 and St-g1 grades vs clustering of St-g2 and St-g3 grades.

${ }^{\mathrm{b}} P$-value after clustering of portal activity 0 and $1 \mathrm{vs}$ clustering of portal activity 2 and 3.

${ }^{\mathrm{c}} P$-value after clustering of lobular necrosis 0 and $1 \mathrm{vs}$ lobular necrosis 2 .

${ }^{\mathrm{d}} P$-value after clustering of portal fibrosis 0 and 1 vs clustering of portal fibrosis 2, 3 and 4 .

${ }^{\mathrm{e}} P$-value after clustering of Mt-g0 and Mt-g1 grades vs Mt-g2 grade.

'mottled' pattern of labeling within the hepatic lobule (Figure 4a). In cases with dramatic reduction of COX subunit I staining, islands of labeled hepatocytes persisted within nearly a totally negative lobule (Figure 4c). On the contrary, COX subunit IV (encoded by nDNA) was unaffected in all 13 cases (Figure 4b), although an inconspicuous labeling heterogeneity was present in cases with dramatic reduction of COX subunit I (Figure 4d).

\section{Relationship between Clinical Findings and Histological Findings}

Table 3 summarizes the clinical, biological, and histological findings according to the normal or decreased liver labeling of COX subunit I. A decreased expression of COX subunit I labeling was never observed in untreated patients. Reduction of the expression in COX subunit I was associated with a significant rise in AST, ALT, and AP in 

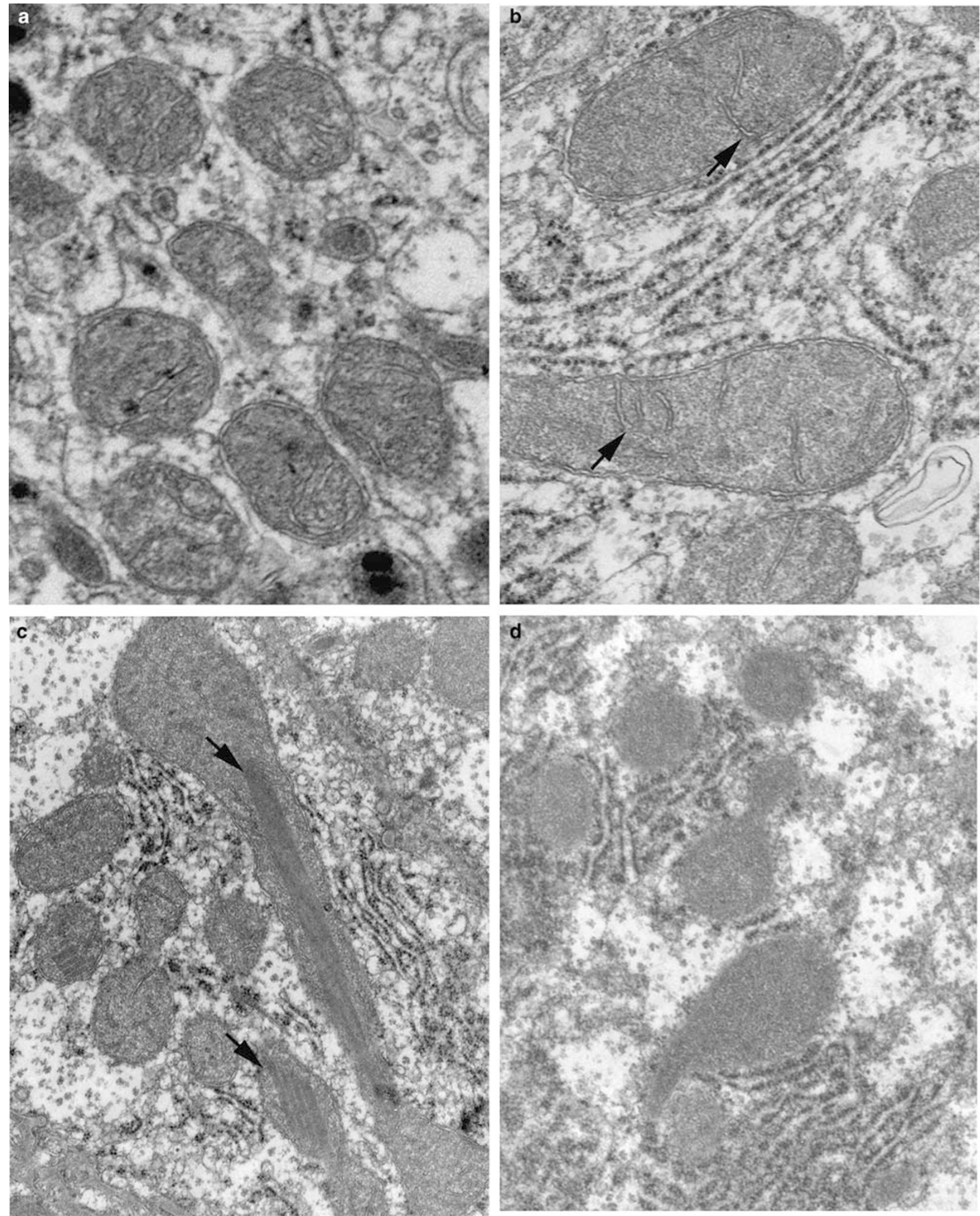

Figure 2 Ultrastructural findings in HIV patients. (a) Normal mitochondria in a HIV-HCV coinfected patient without antiretroviral treatment. (b) Mild mitochondrial defects (Mt-g1) in a monoinfected patient treated with NRTI. Incomplete loss of mitochondrial profiles with persistence of normal profiles (arrows) in nearly all mitochondria. (c) Numerous paracristallin inclusions (arrows) in enlarged mitochondria. (d) Severe mitochondrial alteration (Mt-g2) with almost total loss of mitochondrial profiles and amorphous mitochondrial matrix. 

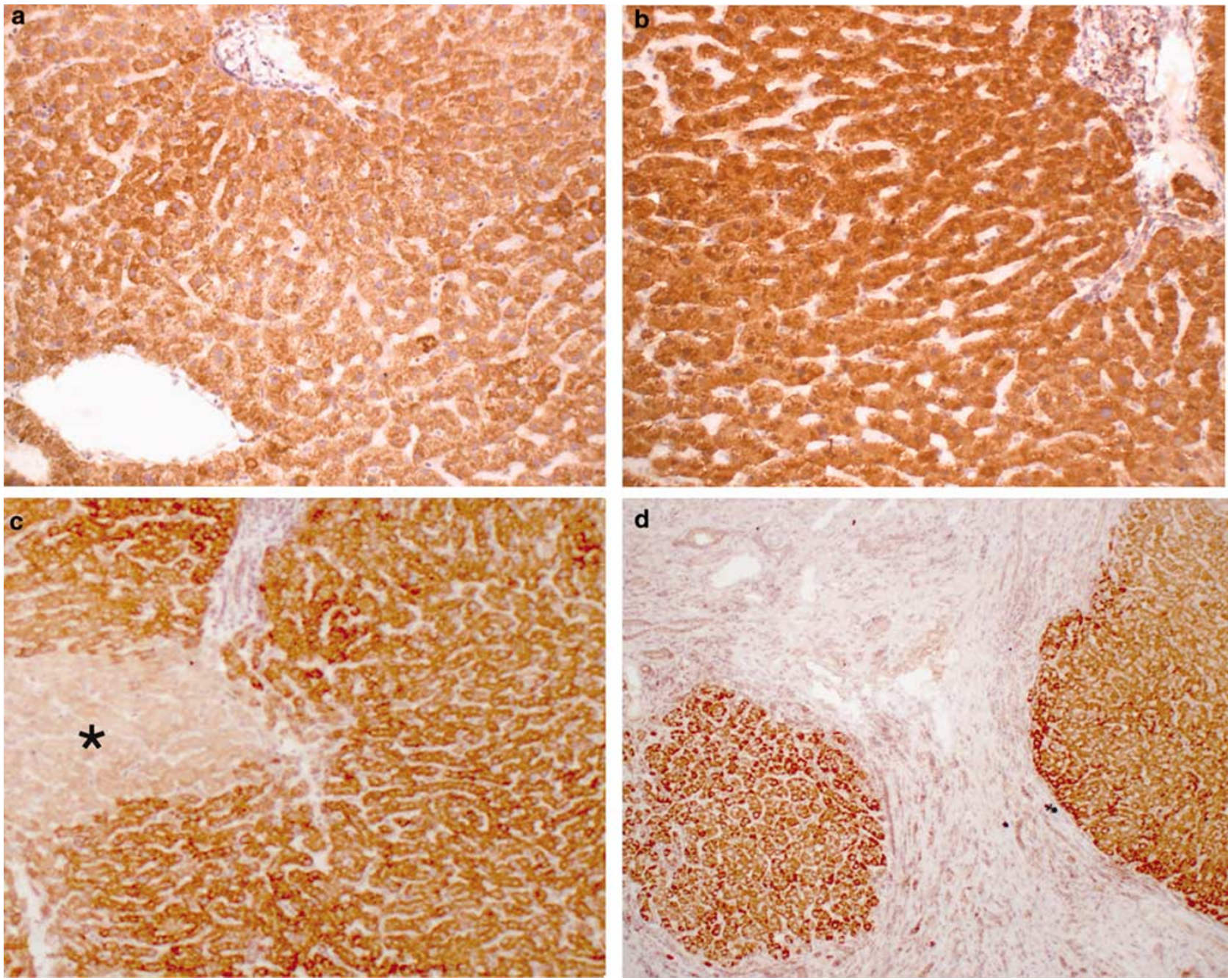

Figure 3 Immunohistochemistry with COX subunit I (a, c, d) and COX subunit IV (b) in a control liver. (a) Homogeneous hepatocyte labeling with COX subunit I antibody in a normal liver surgical biopsy. (b) Homogeneous labeling with COX subunit IV in the same case. (c) Area of decreased labeling with COX subunit I in normal liver $\left({ }^{*}\right)$. (d) COX subunit I staining is maintained with a homogeneous pattern in overt alcoholic cirrhosis.

coinfected patients only. In both mono and coinfected patients, cases with decreased COX subunit I exhibited a rise in the lactates, although not significant in monoinfected patients. Extrahepatic syndrome was more frequently observed in COX subunit I negative patients. Steatosis was more frequent when COX subunit I was decreased. Thus, mean steatosis grade was significantly higher in patients with decreased of COX subunit I, both mono- and coinfected.

Relationship between COX subunit I labeling and ultrastructural data is illustrated in Table 4. Statistical analysis (Yates' $\chi^{2}$ ) after clustering of monoand coinfected patients, and of Mt-g0 and Mt-g1 grades, showed a significant association between decreased COX subunit I and severe mitochondrial abnormalities in EM (Mt-g2). Among the 13 patients with reduction of COX subunit I labeling (nine monoinfected patients and four coinfected patients), 11 patients (85\%) had severe mitochondrial abnorm- alities at EM, but two patients had only minor abnormalities. Normal expression of COX subunit I was associated with normal EM (13 cases), mild mitochondrial abnormalities (26 cases), and severe mitochondrial defects (three cases). EM grade was significantly higher in patients with a decrease in COX subunit I labeling.

Five cases had discordant results between COX subunit I labeling and EM (Table 5) and were detailed as follow: 1/3 out of 14 cases with severe ultrastructural abnormalities were not associated with decreased COX subunit I (case nos. 1-3). The first case (case no. 1) was a monoinfected patient with subnormal liver tests and liver histology. Two coinfected cases were clinically or histologically suggestive of mitochondrial toxicity. In one case (case no. 2), liver histology showed St-g2 steatosis with foci of microvesicular steatosis, hepatocyte ballooning degeneration, and megamitochondria, but lactates were normal. In the other case (case 

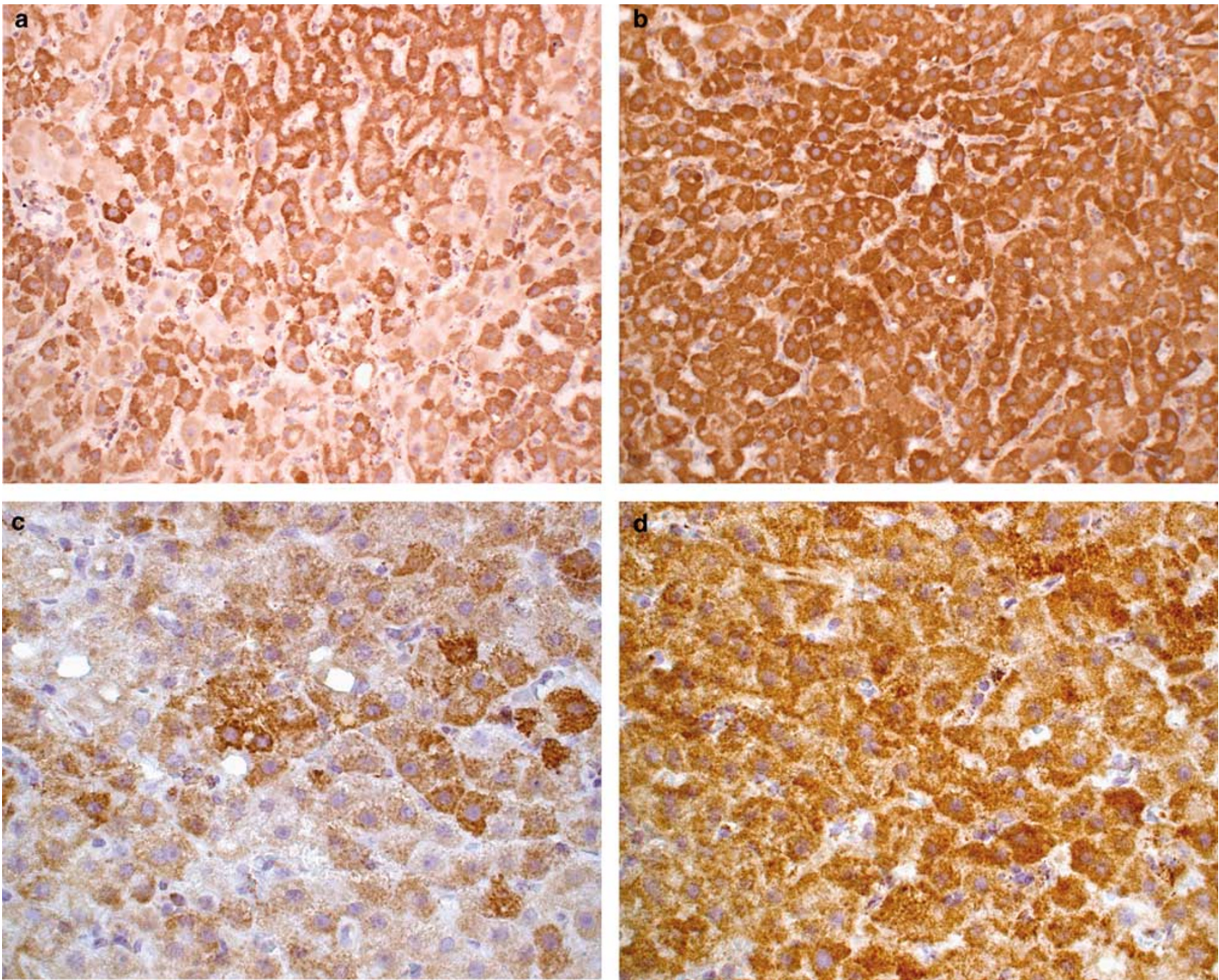

Figure 4 Immunohistochemistry with COX subunit I $(\mathbf{a}, \mathbf{c})$ and COX subunit IV $(\mathbf{b}, \mathbf{d})$ in liver biopsies of HIV patients treated with NRTI. (a) Heterogeneous loss of COX subunit I hepatocyte staining in a monoinfected patient. (b) Normal labeling with COX subunit IV in the same patient as (a). (c) Severe loss of COX subunit I in a coinfected patient, with persistence of islands of labeled cells. (d) Maintained expression of COX subunit IV in a inconspicuous inhomogeneous pattern in the same patient as (c).

no. 3), lactates were $3.2 \mathrm{mmol} / \mathrm{l}$ but with isolated mild steatosis St-g1 on liver biopsy; 2/2 patients with minor (Mt-g1) abnormalities had reduced labeling of COX subunit I associated. Case no. 4 was a monoinfected patient with steatosis St-g3, hepatocyte ballooning degeneration, and megamitochondria at histology, but lactates were normal. Case no. 5 was a coinfected patient with high lactates, moderate steatosis, and severely increased AT levels.

Considering prospectively enrolled HIV-HCV coinfected patients alone, steatosis was present in $64 \%$ of cases. Steatosis was not significantly associated with genotype 3, METAVIR activity or fibrosis score, EM findings or COX subunit I labeling. The distribution of METAVIR activity and fibrosis scores was then assessed according to COX subunit I labeling and EM grade. Mean fibrosis score was significantly elevated in patients with decreased COX subunit I labeling ( $F$ score: $3+/-0.6$ in decreased COX subunit I patients vs $1.6+/-0.2$ in normal COX subunit I patients, $P<0.05)$ and in severe mitochondrial abnormalities at EM (A score: $2.6+/-0.4$ in Mt-g2 patients vs $1.6+/-0.2$ in Mt-g0 and $1.6+/-0.3$ in Mt-g1, $P<001)$. Mean activity score was similar in all groups.

\section{Discussion}

In the present study, the immunohistochemical detection of respiratory chain protein COX subunits I and IV in liver biopsies from HIV patients was evaluated for the diagnosis of NRTI-associated toxicity. Our main result is that decreased expression of mt-DNA encoded COX subunit I is associated with severe ultrastructural mitochondriopathy. Thus, COX subunit I labeling and EM together classify $90 \%$ of our patients into: (i) patients with 'overt mitochondrial cytopathy' characterized by 
Table 3 Distribution of clinical, biological, and histopathological findings according to COX subunit I immunolabeling

\begin{tabular}{|c|c|c|c|c|c|}
\hline & \multicolumn{4}{|c|}{ COX subunit I } & \multirow[t]{3}{*}{$\mathrm{P}$} \\
\hline & \multicolumn{2}{|r|}{ Normal } & \multicolumn{2}{|c|}{ Decreased } & \\
\hline & $H I V(\mathrm{~N}=7)$ & $H I V-H C V(\mathrm{~N}=35)$ & $H I V(\mathrm{~N}=9$ & $H I V-H C V(\mathrm{~N}=4)$ & \\
\hline Antiretroviral treatment: $N$ & 7 & 26 & 9 & 4 & $\mathrm{NS}^{\mathrm{a}}$ \\
\hline Extrahepatic syndrome: $N$ & 3 & 11 & 6 & 4 & $<0.001^{\mathrm{a}}$ \\
\hline \multicolumn{6}{|l|}{ Biology } \\
\hline AST (IU/l): mean \pm s.e.m. & $68 \pm 11$ & $56 \pm 6$ & $78 \pm 45$ & $454 \pm 280$ & $\mathrm{NS}^{\mathrm{b}},<0.001^{\mathrm{c}}$ \\
\hline ALT (IU/l): mean \pm s.e.m. & $95 \pm 31$ & $75 \pm 9$ & $110 \pm 18$ & $444 \pm 241$ & $\mathrm{NS}^{\mathrm{b}},<0.001^{\mathrm{c}}$ \\
\hline AP (IU/l): mean \pm s.e.m. & $100 \pm 19$ & $69 \pm 3$ & $100 \pm 33$ & $93 \pm 9$ & $\mathrm{NS}^{\mathrm{b}},<0.001^{\mathrm{c}}$ \\
\hline$\gamma \mathrm{GT}(\mathrm{IU} / \mathrm{l}):$ mean \pm s.e.m. & $223 \pm 60$ & $120 \pm 24$ & $246 \pm 83$ & $274 \pm 74$ & $\mathrm{NS}^{\mathrm{b}}, \mathrm{NS}^{\mathrm{c}}$ \\
\hline Lactates $(\mathrm{mmol} / \mathrm{l})$ : mean \pm s.e.m. & $1.8 \pm 0.4$ & $1.4 \pm 0.1$ & $2.9 \pm 0.4$ & $2.4 \pm 0.6$ & $\mathrm{NS}^{\mathrm{b}},<0.001^{\mathrm{c}}$ \\
\hline \multicolumn{6}{|l|}{ Steatosis } \\
\hline Absent (St-g0): $N$ & 3 & 14 & 0 & 0 & $<0.001^{\mathrm{d}}$ \\
\hline Mild (St-g1): $N$ & 3 & 18 & 1 & 1 & \\
\hline Moderate (Mt-g2): $N$ & 0 & 3 & 3 & 3 & \\
\hline Severe (Mt-g3): $N$ & 1 & 0 & 5 & 0 & \\
\hline St-grade: mean \pm s.e.m. & $0.8 \pm 0.4$ & $0.7 \pm 0.1$ & $2.4 \pm 0.2$ & $1.8 \pm 0.2$ & $<0.001^{\mathrm{b}},<0.001^{\mathrm{c}}$ \\
\hline Microvesicular steatosis: $N$ & 1 & $\frac{-1}{1}$ & 4 & $\frac{1}{1}$ & $<0.001^{\mathrm{e}}$ \\
\hline $\begin{array}{l}\text { Hepatocyte clarification, megamitochondria, } \\
\text { Mallory: } N\end{array}$ & 3 & 7 & 9 & 2 & \\
\hline
\end{tabular}

AST: aspartate aminotransferase, ALT: alanine aminotransferase, AP: alkalin phosphatase, $\gamma$ GT: gamma glutamyl transferase. Statistical anlysis with comparison between COX subunit I labeling 'normal' vs 'decreased' groups.

${ }^{\mathrm{a}} P$-value after clustering of HIV and HIV-HCV groups.

${ }^{\mathrm{b}} P$-value for HIV patients.

${ }^{\mathrm{c}} P$-value for HIV-HCV patients.

${ }^{\mathrm{d}} P$-value after clustering of HIV and HIV-HCV patients, clustering of St-g0 and St-g1 grades vs clustering of St-g2 and St-g3 grades.

${ }^{\mathrm{e}} \mathrm{P}$-value after clustering of HIV and HIV-HCV groups and clustering of microvesicular steatosis and hepatocyte clarification, megamitochondria, Mallory bodies.

Table 4 Distribution of electron microscopy mitochondrial lesion grades according to COX subunit I labeling patterns

\begin{tabular}{|c|c|c|c|c|c|}
\hline & \multicolumn{4}{|c|}{ COX subunit I } & \multirow[t]{3}{*}{$\mathrm{P}$} \\
\hline & \multicolumn{2}{|c|}{ Normal } & \multicolumn{2}{|c|}{ Decreased } & \\
\hline & $H I V(\mathrm{~N}=7)$ & $H I V-H C V(\mathrm{~N}=35)$ & $H I V(\mathrm{~N}=9)$ & $H I-H C V(\mathrm{~N}=4)$ & \\
\hline \multicolumn{6}{|c|}{ EM mitochondrial abnormalities } \\
\hline Absent (Mt-g0): N & 1 & 12 & 0 & 0 & $<0.001^{\mathrm{a}}$ \\
\hline Mild (Mt-g1): N & 5 & 21 & 1 & 1 & \\
\hline Severe (Mt-g2): N & 1 & 2 & 8 & 3 & \\
\hline Mt-grade: mean \pm s.e.m. & $1.0 \pm 0.2$ & $0.7 \pm 0.1$ & $1.9 \pm 0.1$ & $1.7 \pm 0.2$ & $<0.001^{\mathrm{b}},<0.001^{\mathrm{c}}$ \\
\hline
\end{tabular}

Statistical analysis with comparison between COX subunit I labeling 'normal' vs 'decreased' groups.

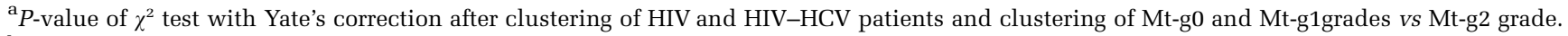

${ }^{\mathrm{b}} P$-value for HIV patients.

${ }^{\mathrm{c}} P$ value for HIV-HCV patients.

decreased COX subunit I labeling and severe ultrastructural mitochondriopathy; (ii) patients with 'isolated mild ultrastructural mitochondrial abnormalities' characterized by normal COX subunit I labeling and mild ultrastructural abnormalities; (iii) patients with 'no mitochondrial structural damage' characterized by normal COX subunit I labeling and EM. However, the remaining five cases (out of 55) represent discrepancies between COX labeling and EM: decrease in COX subunit I was lacking in three cases with severe EM abnormalities, and was detected in two cases with only mild mitochondrial EM lesions. Such discrepancies might be due to: (1) sampling limitation of EM, since ultrastructural examination is restricted to a very small amount of tissue. Furthermore, the reduction COX subunit I labeling is highly variable between adjacent hepatocytes in the same liver 
Table 5 Characteristics of patients with discrepancy between electron microscopy and COX subunit I labeling

\begin{tabular}{|c|c|c|c|c|c|}
\hline Case number & 1 & 2 & 3 & 4 & 5 \\
\hline EM grade & Mt-g2 & Mt-g2 & Mt-g2 & Mt-g1 & Mt-g1 \\
\hline COX subunit I & Normal & Normal & Normal & Decreased & Decreased \\
\hline Group & HIV treated & HIV-HCV treated & HIV-HCV treated & HIV treated & HIV-HCV treated \\
\hline Treatment & 2 NRTI, PI & 2 NRTI, NNRTI & 2 NRTI, PI & 2 NRTI & 2 NRTI, NNRTI \\
\hline Aminotransferase (IU/l) & AST: 51 ALT: 63 & AST: 57 ALT: 83 & AST: 58 ALT: 149 & AST: 88 ALT: 185 & AST: 195 ALT: 319 \\
\hline Lactates (mmol/l) & 1.9 & 1.3 & 3.2 & 1.4 & 3.8 \\
\hline Extrahepatic sd & + & - & - & - & + \\
\hline Steatosis grade & St-g0 & St-g2 & St-g1 & St-g3 & St-g2 \\
\hline Histology & - & $\mathrm{HB}, \mathrm{MM}, \mathrm{MS}$ & - & $\mathrm{HB}, \mathrm{MM}, \mathrm{MB}$ & - \\
\hline
\end{tabular}

AST: aspartate aminotransferase, ALT: alanine aminotransferase, HB: hepatocyte balloonning/clarification, MM: Mallory bodies, MM: megamitochondria, MS: microvesicular steatosis.

biopsy (see Figure 4a,c). Such heterogeneity may worsen the problem of sampling in EM; (2) differences in the sensitivity of the two techniques for the detection of mitochondrial damage. It is likely that COX subunit I labeling is less sensitive than EM for the diagnosis of structural abnormalities of the mitochondria.

As two groups of patients are characterized, one with 'isolated mild ultrastructural mitochondrial abnormalities' and the other with 'overt mitochondrial cytopathy', our results probably reflect the pathogenesis of NRTI-associated mitochondriopathy in HIV/AIDS patients. ${ }^{7,8}$ In this 'two-hit' injury model, mitochondrial injury is multifactorial and may be explained by the cumulative effects of mitochondrial insults depending on host factors, drugs, oxidative stress, etc. ${ }^{18-20}$ It is likely that decreased COX subunit I labeling and severe ultrastructural alteration correspond to profound and clinically relevant drug-induced liver toxicity, that was observed in $50 \%$ of HIV monoinfected patients and in $13 \%$ of coinfected patients. In monoinfected patients, clinical features alone were unable to diagnose severe mitochondriopathy, whereas, drug toxicity strongly impaired liver tests and lactate levels in coinfected patients. As expected, macrovacuolar and microvesicular steatosis were associated with decreased COX subunit I labeling and severe ultrastructural mitochondriopathy in both groups. The 'two-hit' injury model of mitochondrial injury may also explain our cases with isolated EM abnormalities. In these cases, although clinically relevant drug toxicity is unlikely, mitochondrial ultrastructural alterations may be explained by the cumulative deleterious effects of various origins. HIV infection per se has been associated with mitochondrial dysfunction. ${ }^{21,22}$ Alteration of mitochondrial structure have also been reported in HCV monoinfected patients, together with induction of the oxidative stress and steatosis. ${ }^{23-27}$ As a matter of fact, Bauerle et $a l^{28}$ have recently measured liver mtDNA of HCV monoinfected patients and in HIV-HCV patients, before any antiretroviral treatment. Liver mtDNA levels was decreased in both HCV and HIV-HCV patients as compared to normal controls, and the mtDNA depletion was more pronounced in HIV-HCV patients.

Finally, the value of COX subunit I labeling should be addressed by taking into account the considerable efforts that have been made within the last few years to improve the diagnosis of NRTIinduced mitochondrial cytopathy in HIV/AIDS patients. Recent studies have made attempts to demonstrate the usefulness of various biochemical, molecular, and functional mitochondrial tests on peripheral leukocytes for the clinical practice. ${ }^{12-15}$ Although several authors have reported that peripheral leukocytes mtDNA/nDNA ratio determined by RT-PCR was decreased in symptomatic hyperlactacidemia, ${ }^{12,13}$ others have observed discordant results between peripheral mtDNA measurement, functional mitochondrial evaluation and clinical parameters that is lactates. ${ }^{14,15}$ That mitochondrial assessment in peripheral leukocytes reflects a target organ toxicity, that is, the liver toxicity has not been clearly demonstrated. Thus, Vittecoq et $a{ }^{29}$ have reported a dissociation between mitochondrial evaluation in muscular biopsies and in peripheral leukocytes. Interestingly, Walker et $a l^{30}$ reported a profound liver mtDNA depletion in patients treated with ddC and ddI, both highly toxic NRTI in vitro. mtDNA was also decreased in patients with elevated lactates.

In summary, COX subunit I labeling in liver biopsies may be a valuable tool for the diagnosis of NRTI-induced mitochondrial cytopathy in HIV patients with clinically low-grade hepatotoxicity. Decreased expression of COX subunit I that is associated with severe ultrastructural abnormalities defines a group of overt NRTI-related mitochondriopathy. Our results also raise the question of the clinical significance of isolated mild ultrastructural mitochondrial abnormalities without alteration of COX subunit I expression. The consequences of low grade but long lasting mitochondrial dysfunction, together with secondary oxidative stress on liver parenchyma are currently unknown and remain speculative ${ }^{27}$ and thus warrant further investigation. 


\section{Acknowledgements}

This work was supported by grants from the Société Française de Pathologie (SFP) and from the Agence Nationale Recherche sur le SIDA (ANRS) 2000/151.

\section{References}

1 Schreider MF, Gauge SJ, Williams CM, et al. Patterns of the hazard of death after AIDS through the evolution of antiretroviral therapy: 1984-2004. Aids 2005;19:20092018.

2 Rosenthal E, Poiree M, Pradier C, et al. Mortality due to hepatitis C-related liver disease in HIV-infected patients in France (MORTAVIC 2001 study). Aids 2003; 17:1803-1809.

3 Pol S, Vallet-Pichard A, Fontaine H. Hepatitis C and HIV co-infection at the era of HAART. J of Viral Hepatitis 2002;9:1-8.

4 Allory Y, Charlotte F, Benhamou Y, et al. Impact of human immunodeficiency virus infection on the histological features of chronic hepatitis C: a casecontrol study. The MULTIVIRC group. Hum Pathol 2000;31:69-74.

5 Rullier A, Trimoulet P, Neau D, et al. Fibrosis is worse in HIV-HCV patients with low-level immunodepression referred for HCV treatment than in HCV-matched patients. Hum Pathol 2004;35:1088-1094.

6 Spengler U, Lichterfeld M, Rockstroh JK. Antiretroviral drug toxicity-a challenge for the hepatologist? J Hepatol 2002;36:283-294.

7 Cossarizza A, Moyle G. Antiretroviral nucleoside and nucleotide analogues and mitochondria. Aids 2004;18: 137-151.

8 Lewis W, Day BJ, Copeland WC. Mitochondrial toxicity of NRTI antiviral drugs: an integrated cellular perspective. Nat Rev Drug Discov 2003;2:812-822.

9 Ogedegbe AE, Thomas DL, Diehl AM. Hyperlactataemia syndromes associated with HIV therapy. Lancet Infect Dis 2003;3:329-337.

10 Van Huyen JP, Landau A, Piketty C, et al. Toxic effects of nucleoside reverse transcriptase inhibitors on the liver. Value of electron microscopy analysis for the diagnosis of mitochondrial cytopathy. Am J Clin Pathol 2003;119:546-555.

11 Batisse D, Van Huyen JP, Piketty C, et al. Severe liver mitochondriopathy with normal liver histology and normal lactate levels in patients receiving nucleoside analogues. Aids 2002;16:2370-2371.

12 Cote HC, Brumme ZL, Craib KJ, et al. Changes in mitochondrial DNA as a marker of nucleoside toxicity in HIV-infected patients. N Engl J Med 2002;346:811-820.

13 Reiss P, Casula M, de Ronde A, et al. Greater and more rapid depletion of mitochondrial DNA in blood of patients treated with dual (zidovudine+didanosine or zidovudine+zalcitabine) vs single (zidovudine) nucleoside reverse transcriptase inhibitors. HIV Med 2004;5:11-14.

14 Lopez S, Miro O, Martinez E, et al. Mitochondrial effects of antiretroviral therapies in asymptomatic patients. Antivir Ther 2004;9:47-55.
15 Chiappini F, Teicher E, Saffroy R, et al. Prospective evaluation of blood concentration of mitochondrial DNA as a marker of toxicity in 157 consecutively recruited untreated or HAART-treated HIV-positive patients. Lab Invest 2004;84:908-914.

16 Tanji N, Tanji K, Kambham N, et al. Adefovir nephrotoxicity: Possible role of mitochondrial DNA depletion. Hum Pathol 2001;32:734-740.

17 Bedossa P, Poynard T. An algorithm for the grading of activity in chronic hepatitis C. The METAVIR Cooperative Study Group. Hepatology 1996;24: 289-293.

18 Verucchi G, Calza L, Biagetti C, et al. Ultrastructural liver mitochondrial abnormalities in HIV/HCV-coinfected patients receiving antiretroviral therapy. J Acquir Immune Defic Syndr 2004;35:326-328.

19 Laguno M, Milinkovic A, de Luzzari E, et al. Incidence and risk factors for mitochondrial toxicity in treated HIV/HCV-coinfected patients. Antivir Ther 2005;10: 423-429.

20 Bani-Sadr F, Carrat F, Pol S, et al. Risk factors for symptomatic mitochondrial toxicity in HIV/hepatitis C virus-coinfected patients during interferon plus ribavirin-based therapy. J Acquir Immune Defic Syndr 2005;40:47-52.

21 Miro O, Lopez S, Martinez E, et al. Mitochondrial effects of HIV infection on the peripheral blood mononuclear cells of HIV-infected patients who were never treated with antiretrovirals. Clin Infect Dis 2004;39:710-716.

22 Miura T, Goto M, Hosoya $\mathrm{N}$, et al. Depletion of mitochondrial DNA in HIV-1-infected patients and its amelioration by antiretroviral therapy. J Med Virol 2003;70:497-505.

23 Barbaro G, Di Lorenzo G, Asti A, et al. Hepatocellular mitochondrial alterations in patients with chronic hepatitis C: ultrastructural and biochemical findings. Am J Gastroenterol 1999;94:2198-2205.

24 Rust C, Gores GJ. Does hepatitis C cause liver injury by pathways associated with mitochondrial dysfunction? Am J Gastroenterol 1999;94:2003-2005.

25 Pessayre D, Berson A, Fromenty B, et al. Mitochondria in steatohepatitis. Semin Liver Dis 2001;21: 57-69.

26 Asselah T, Boyer N, Marcellin P. Steatosis in hepatitis C: What does it mean? Curr Hepat Rep 2003; 2:137-144

27 de Mendoza C, Soriano V. The role of hepatitis $\mathrm{C}$ virus (HCV) in mitochondrial DNA damage in HIV-HCV coinfected individuals. Antivir Ther 2005;10(supple 2):M109-M115.

28 Bauerle J, Laguno M, Mauss S, et al. Mitochondrial DNA depletion in liver tissue of patients infected with hepatitis $\mathrm{C}$ virus: contributing effect of HIV infection? HIV Med 2005;6:135-139.

29 Vittecoq D, Jardel C, Barthelemy C, et al. Mitochondrial damage associated with long-term antiretroviral treatment: associated alteration or causal disorder? J Acquir Immune Defic Syndr 2002;31:299-308.

30 Walker UA, Bauerle J, Laguno M, et al. Depletion of mitochondrial DNA in liver under antiretroviral therapy with didanosine, stavudine, or zalcitabine. Hepatology 2004;39:311-317. 